\title{
An Approach to Assessing Unidimensionality Revisited
}

\author{
Iระaต 1. Beำ \\ Edincational Tosting Sarvice
}

\begin{abstract}
A reanalysis of data from Hambleton and Rovinelli (1986) argues that the methods suggested by Bejar (1980) are a valuable descriptive tool for assessing the unidimensionality assumption when a priori information is available about possible response factors. Index terms: achievement testing, item response theory, unidimensionality.
\end{abstract}

Hambleton and Rovinelli (1986) recently argued that Bejar's (1980) method to assess the dimensionality of a set of items was insensitive to the multidimensionality in the data in all but one of four of their analyses. However, Hambleton and Rovinelli equated the "Bejar method" with computing a correlation coefficient between two sets of item parameter estimates. In reality, Bejar proposed examining the deviations from a theoretical axis in conjunction with an analysis of residuals from a one-factor model fitted to a covariance matrix of subscores.

Specifically, Bejar suggested:

1. Identifying subsets of items that tap different psychological dimensions, or in the case of an achievement test, a different knowledge base;

2. Fitting an item response theory (IRT) model separately for each subset as well as for all items together;

APPLHED PSYCHOLOGICAL MEASUREMENT

Vol.12, No.4, December 1988, pp. 377-379

(c) Copyright 1988 Applied Psychological Measurement Inc. 0146-6216/88/040377-03\$1.40
3. Plotting the subset-based item difficulty parameter estimates against the total-based estimates for each subset of items;

4. Determining if unidimensionality holds by observing whether the two sets of estimates fall on an axis with a slope of 1 and an intercept of 0 , which should occur if the subset-based and total-based estimation is performed on the same sample or random samples from the same population; and

5. Interpreting deviations from that theoretical axis as deviations from unidimensionality.

Bejar also proposed performing a factor analysis of ability estimates based on the subsets and fitting a one-factor model to it. Again, deviations from the one-factor hypothesis would provide evidence against unidimensionality. In short, Bejar proposed two criteria to examine departures from unidimensionality. The correlation between two sets of estimates was not one of them.

\section{Reanalysis}

Hambleton and Rovinelli provided data that allow a reanalysis, namely the two sets of item parameter estimates on several sets of multidimensional data (in their Table 5). The data consisted of five artificially generated datasets of 40 items and 1,500 examinees. One dataset was generated to conform to the unidimensional model. The additional four datasets were generated to contain two 
factors, and varied the number of items belonging to each factor and the correlation between the first and second factors.

The plots of the total and subset-based estimates for the five datasets appear in Figure 1. Figure 1 shows that when unidimensionality holds, the two sets of estimates fall very close to the theoretical axis. For the two-dimensional datasets with equal numbers of items in each dimension, Figures $2 c$ and $2 \mathrm{~d}$ show little departure from the theoretical axis, although there appears to be a slight degree of curvilinearity at the high end. However, for the two datasets with unequal numbers of items in each dimension, Figures $2 \mathrm{a}$ and $2 \mathrm{~b}$ clearly indicate that unidimensionality does not hold, especially for the dataset with a correlation of .60 (Figure 2b). It also
Fi'igure $\mathbb{1}$

Plot of Subset-Based and Total-Based Estimates of Item Difficulty for a One-Dimensional Dataset

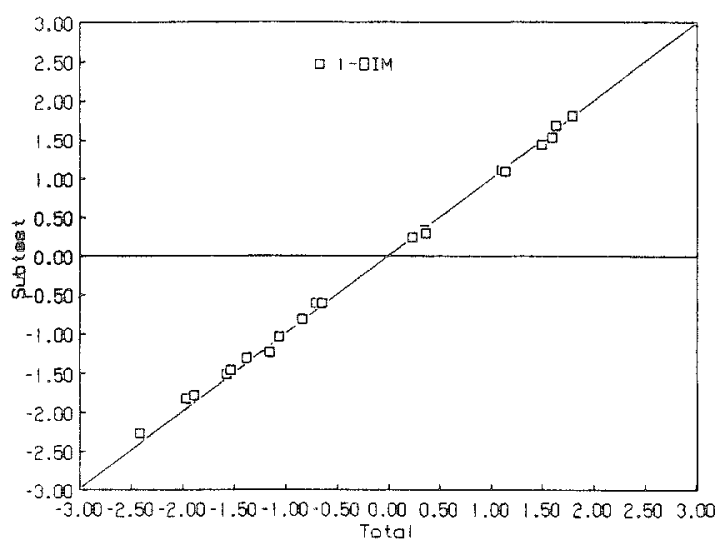

Tignare 2

Plot of Subset-Based and Total-Based Estimates of Item Difficulty for Two-Dimensional Datasets

(a) $r=.10, n_{1}=30, n_{2}=10$

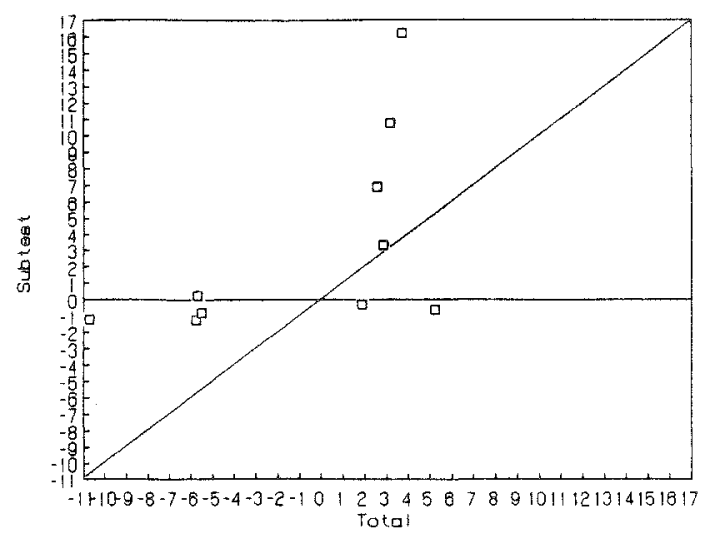

(c) $r=.10, n_{1}=20, n_{2}=20$

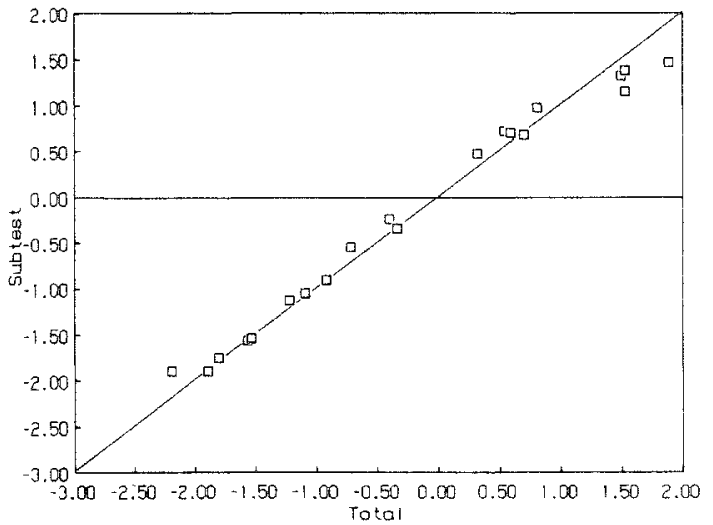

(b) $r=.60, n_{1}=30, n_{2}=10$

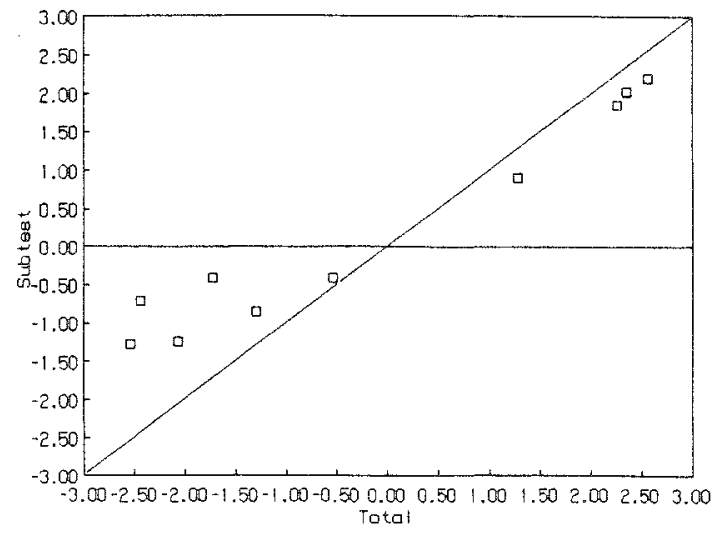

(d) $r=.60, n_{1}=20, n_{2}=20$

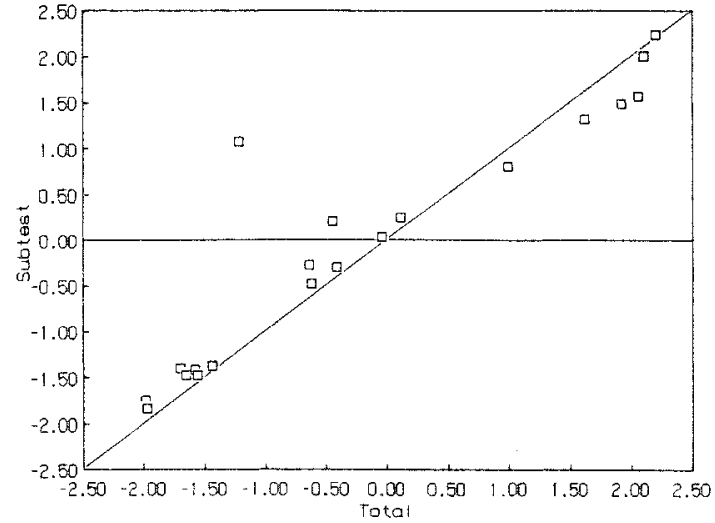


seems that unidimensionality does not hold for the dataset with a correlation of .10 (Figure $2 \mathrm{a}$ ), but this could have been due to poor estimation of item parameters because LOGIST converged too soon.

\section{Conclusions}

Although noticeable departures existed from the theoretical axis for all the multidimensional datasets, this reanalysis suggests that examining the bivariate plots of the difficulty parameter estimates is not as powerful in detecting departures from unidimensionality when equal numbers of items exist in each dimension. Nevertheless, as a descriptive tool, the method remains valuable (Henning, Hudson, \& Tumer, 1985) when different sets of items may call for different configurations of abilities.

\section{References}

Bejar, 1. I. (1980). A procedure for investigating the unidimensionality of achievement tests based on item parameter estimates. Joumal of Educational Measurement, 17, 283-296.

Hambleton, R. K., \& Rovinelli, R. J. (1986). Assessing the dimensionality of a set of test items. Applied Psychological Measurement, 10, 287-302.

Henning, G., Hudson, T., \& Tumer, J. (1985). Item response theory and the assumption of unidimensionality for language tests. Language Testing, 2, 141154.

\section{Auter}

Send requests for reprints or further information to Isaac I. Bejar, Educational Testing Service, Princeton NII 08541, U.S.A. 